\title{
Relación entre la práctica de actividad físico-deportiva y el consumo de alcohol de los ciudadanos de más de 14 años
}

\author{
Alfonso Valero Valenzuela*; Francisco Ruiz Juan**; Ma Elena García Montes**; \\ antonio Granero Gallegos***; Ana María Martínez Rodríguez**** \\ * Universidad de Murcia. Profesor del Área de Educación Física y Deportiva. \\ * Universidad de Murcia. Profesor/a del Área de Educación Física y Deportiva. \\ *** Universidad de Almería. Profesor del Área de Didáctica de la Expresión Corporal. \\ **** Universidad de Jaén. Profesora del Departamento de Estadística e Investigación Operativa.

\section{RESUMEN}

El objetivo de la presente investigación es conocer la posible relación que puede existir entre el consumo de alcohol que realiza la población con las diferentes variables sociodemográficas, así como la influencia que pueda ejercer la práctica físico-deportiva en la creación de unos hábitos de vida saludables. La muestra está compuesta por 1102 sujetos pertenecientes a la provincia de Almería, mayores de 14 años, a los que se les pasa el cuestionario denominado "hábitos físico deportivos y estilos de vida", seleccionando los apartados de hábitos físicodeportivos de tiempo libre, la percepción de la condición física y del estado de salud y los hábitos de consumo de alcohol, relacionados con las variables actitudinales, comportamentales y sociodemográficas. Se ha empleado una herramienta innovadora para el análisis de los datos dentro del ámbito de la actividad física como son las redes bayesianas, destacando una relación positiva del consumo de alcohol con la práctica de actividad físico-deportiva, al ser las personas activas con interés por la actividad físico-deportiva, que poseen una buena percepción de su salud y condición física, las que realizan un mayor consumo habitual y ocasional de esta sustancia. El perfil de ciudadano que no consume alcohol son mujeres, con más de 44 años, sin estudios y amas de casa, que poseen una mala percepción de su salud, que no practican actividades físico-deportivas, mientras que las poblaciones en riesgo para la salud, son los varones que tienen entre 15 y 44 años, que poseen estudios secundarios o con una licenciatura y que trabajan en el sector público o privado.

Palabras clave: Alcohol, actividad físico-deportiva, hábitos de

\section{ABSTRACT}

The aim of the current research is to ascertain any possible relationship that could exist between the alcohol consumption of the population and several sociodemographic variables, in addition to the influence of physical exercise/sports on the creation of healthy lifestyle habits. The sample comprised 1102 subjects from Almeria Spain, over 14 years of age, to whom we administered the "physical exercise/sporting habits and life styles" questionnaire, selecting the sections on physical exercise/sporting habits in leisure, fitness and state of health perception and alcohol consumption habits, related to attitudes, behavioural and sociodemographic variables. We used an innovative tool for data analysis in the sphere of physical exercise, known as the Bayesian networks, emphasizing a positive relationship between alcohol consumption and physical exercise/sporting activities, it being the active people, with an interest in physical exercise/sports, who have a good perception of their health and physical fitness who are the ones who make habitual and occasional use of this substance. The profile of the individual who does not drink alcohol is female, over 44 years of age, with no studies, housewives who have a poor perception of their health, and who do not do any physical exercise or plays sports, whereas the populations with health risks, are men between 15 and 44 years of age who have secondary school studies or a degree and who work in the public or private sector

Key words: Alcohol, physical-sport activity, life habits, health, life style. 


\section{NTRODUCCIÓN}

E | alcohol forma parte de nuestra vida social y cultural y, en general, se observa una excesiva permisividad en el consumo incluso abusivo de bebidas alcohólicas pero, al mismo tiempo, se rechaza todo lo que suena a alcoholismo. Por un lado, se intenta otorgar a las bebidas alcohólicas un valor nutritivo e incluso protector de la salud (especialmente la cerveza y el vino) y, por otro, se observa un rechazo social al abuso (Pascual, 2002a y b).

Gran parte de los estudios relacionados con la ingesta de alcohol y los problemas que desencadenan se han centrado en la adolescencia (Moral, Rodríguez y Sirvent, 2005; Carrasco, 2004; Portero, Cirne y Mathieu, 2002; Mora-Rios y Natera, 2001). Tanto a nivel nacional (Plan Nacional sobre Drogas, 2005), como a nivel andaluz (Junta de Andalucía, 1997), se ha comprobado la existencia de un elevado consumo semanal especialmente en fines de semana entre los jóvenes. Precisamente, esta conducta de consumo de alcohol en edades tempranas predice problemas relacionados con el alcohol en el adulto joven y su vida posterior, mayor riesgo en el abuso del alcohol y problemas relacionados con la adolescencia e incluso dependencia en la edad adulta hacia este tipo de sustancias (Riala, Hakko, Isohanni, Järvelín y Räsänen, 2004).

En cuanto al sexo, son los hombres quienes realizan un mayor consumo como así se comprueba en estudios tanto a nivel internacional (Simpson, Brehm, Rasmussen, Ramsay y Probst (2002), como a nivel nacional (Plan Nacional sobre Drogas, 2005) y para las distintas comunidades autónomas como la andaluza (Junta de Andalucía, 1997), la valenciana (Balaguer, Pastor y Moreno, 1999) y la madrileña (Hidalgo, Garrido y Hernández, 2000) entre otras.

Otras variables sociodemográficas que pueden estar relacionadas con el consumo de alcohol son el nivel socioeconómico (Denton y Walters, 1999), donde un peor estado de salud se asocia a un menor estatus socioeconómico, si bien, para otras drogas que no sean el alcohol y el tabaco, a mayor nivel económico corresponde un mayor consumo de drogas ilegales (Santo-Domingo, 2002). Además, la actividad principal y los estudios realizados, son otras variables que conviene tener en consideración, ya que tal y como se obtiene en el estudio de la Junta de Andalucía (1997), las personas en paro, y con fracaso escolar coadyuvan a un consumo abusivo del alcohol.

La percepción de riesgo ante la conducta de consumo de alcohol puede ser un indicador indirecto de la evolución presente o futura de la prevalencia de consumo, de forma que si la población percibe que una conducta de consumo puede causar pocos problemas es lógico pensar que pueda existir una tendencia a que el consumo se extienda. En el caso del alcohol se asocia a un menor riesgo que se consuma 5 o 6 cañas o copas los fines de semana a el consumo esporádico de cannabis (Plan Nacional sobre Drogas, 2005), lo que nos lleva a pensar que pueda ser una explicación a por qué según Gómez-Talegón, Prada, Río y Álvarez (2005), España es uno de los países donde el consumo de bebidas alcohólicas y los problemas relacionados con el mismo adquieren una gran importancia.

De entre las variables de protección ante el problema del consumo de alcohol, Martínez y Robles (2001), destacan los valores positivos que pueden ejercer el cuerpo y la vida, apuntándose la actividad física y deportiva, durante el tiempo de ocio, como un elemento protector ante esta conducta. Pate, Heath, Dowda y Trost (1996), en su estudio sobre las asociaciones entre la actividad física y los comportamientos saludables entre los adolescentes obtiene interacciones significativas entre el consumo de alcohol y los factores socioculturales que pueden afectar a la relación entre la actividad física y el consumo de alcohol. En España los estudios Ilevados a cabo por el Plan Nacional sobre Drogas (1998), indican que los jóvenes que realizan actividades culturales y deportivas son menos consumidores frente a aquellos que suelen salir a la calle con sus amigos, donde beber se convierte en una necesidad para socializarse y adquiere una función integradora. En esta línea, un estudio llevado a cabo por Pastor, Balaguer y García-Merita (2006), con adolescentes de entre 15 y 18 años en la comunidad valenciana, concluye que la competencia deportiva ejerce una influencia indirecta sobre las conductas de salud, actuando la participación deportiva como variable mediadora en esta relación.

Sin embargo, estudios como el de Bourdeaudhuij y Van Oost (1999) obtienen que no hay resultados concluyentes sobre la actividad física durante el tiempo de ocio como discriminador entre los subgrupos de salud y no saludables. Resultados en la misma línea han obtenido Paavola, Vartiainen y Haukkala (2004), quienes no han podido hallar una asociación entre el consumo de alcohol y la actividad física y que coinciden con los resultados ya arrojados décadas atrás por Blair, Jacobs y Powell (1985).

El objetivo de la presente investigación es conocer la posible relación que puede existir entre el consumo de alcohol que realiza la población almeriense con las diferentes variables sociodemográficas, así como la posible influencia que puede ejercer la práctica físico-deportiva en la creación de unos hábitos de vida saludables. 


\section{MÉTODOS}

\section{Población y muestra}

Este estudio descriptivo y correlacional se ha llevado a cabo en la provincia de Almería con los ciudadanos mayores de 14 años. Los datos de la población fueron facilitados por el Instituto Nacional de Estadística (INE), que ascendía a 512.843 habitantes siendo, la cifra de almerienses mayores de 14 años de 416.973 habitantes, de los cuales 207.013 eran varones y 209.960 mujeres.

La población se ha distribuido por estratos, atendiendo al número de habitantes por municipio (más de 50.000 habitantes, de 20.001 a 50.000 habitantes, de 10.001 a 20.000 habitantes, de 5.001 a 10.000 habitantes, de 2.001 a 5.000 habitantes y menos de 2.001 habitantes), a los municipios, distritos, secciones censales, sexo y edad (grupos quinquenales) (tabla 1). lizando entrevistas personales mediante cuestionario estandarizado denominado "Hábitos físico-deportivos y estilos de vida", al cuál se les pasaron las correspondientes pruebas de validez de contenido y de constructo, teniendo en cuenta que lo que se pretende medir son conductas, comportamientos y opiniones expresadas por cada uno de los sujetos de la población objeto de estudio. Se ha realizado un examen exhaustivo y sistemático del contenido del cuestionario, es decir, de los ítems de la prueba para determinar si comprende una muestra relevante y representativa de todo el contenido que ha de medirse, del objetivo que pretende, teniendo en cuenta las opiniones de expertos en sociometría, psicometría y estadística y la referencia de autores como Buendía (1998), García Ferrando (2000) y Martínez (1995). Además, dadas las características de variabilidad del tipo de muestra, se ha constatado la fiabilidad del cuestionario mediante los cuatro estudios piloto, en condiciones reales, teniendo en cuenta en todo momento las sugeren-

Tabla 1. Tamaño de la muestra según el número de habitantes por municipio, grupos de edad y sexo.

\begin{tabular}{|c|c|c|c|c|c|c|c|c|c|c|c|c|c|}
\hline \multirow[b]{2}{*}{$\begin{array}{c}\text { Estrato por } \mathbf{n}^{\circ} \\
\text { habitantes }\end{array}$} & \multicolumn{4}{|c|}{ Varones } & \multicolumn{4}{|c|}{ Mujeres } & \multicolumn{5}{|c|}{ TOTAL } \\
\hline & 15 a 29 & 30 a 44 & 45 a 59 & + de 59 & 15 a 29 & 30 a 44 & 45 a 59 & + de 59 & 15 a 29 & 30 a 44 & 45 a 59 & + de 59 & Total \\
\hline+50.000 & 79 & 67 & 40 & 36 & 74 & 72 & 47 & 56 & 153 & 139 & 87 & 92 & 471 \\
\hline $20.001-50.000$ & 20 & 25 & 10 & 12 & 24 & 21 & 11 & 8 & 44 & 46 & 21 & 20 & 131 \\
\hline $10.001-20.000$ & 18 & 19 & 9 & 15 & 22 & 16 & 12 & 10 & 40 & 35 & 21 & 25 & 121 \\
\hline $5.001-10.000$ & 23 & 25 & 12 & 21 & 29 & 23 & 12 & 14 & 52 & 48 & 24 & 35 & 159 \\
\hline $2.001-5.000$ & 15 & 16 & 10 & 16 & 16 & 14 & 14 & 18 & 31 & 30 & 24 & 34 & 119 \\
\hline-2.001 & 13 & 12 & 8 & 16 & 13 & 11 & 10 & 18 & 26 & 23 & 18 & 34 & 101 \\
\hline TOTAL & 164 & 89 & 116 & 178 & 157 & 106 & 124 & 164 & 346 & 321 & 195 & 240 & 1102 \\
\hline
\end{tabular}

Se ha aplicado un muestreo polietápico por afijación proporcional, que supone plantear decisiones muestrales en distintas etapas, recurriendo a dos tipos de muestreo: el aleatorio estratificado y el aleatorio por conglomerados (en el estrato municipios), culminando la selección del sujeto por rutas aleatorias en cada área geográfica muestral. La muestra final ha sido de un total de 1.102 sujetos, 541 varones y 561 mujeres, obteniendo una validez en los resultados con un margen de error muestral del $\pm 3 \%$ y un nivel de confianza del $95.5 \%$.

\section{Técnica de obtención de datos, instrumento y variables}

La obtención de datos se ha llevado a cabo con metodología cuantitativa a través de encuestas, rea- cias y aportaciones surgidas en el proceso, así como las realizadas por los expertos que colaboraron en la investigación (Ruiz Juan y García Montes, 2005).

De los distintos bloques temáticos de los que está compuesto el cuestionario, para este artículo se han seleccionado aquellos apartados relacionados con tres grandes grupos de variables que contemplan esta investigación como son las variables actitudinales, dentro de las que se ha distinguido entre interés por la práctica de actividad físico-deportiva, (alto y bajo interés), autopercepción de salud y condición física (buena, regular y mala) y valoración del consumo del alcohol (mucho, poco y nada). Otro bloque de variables son las comportamentales, en las que se halla la práctica de actividad físico-deportiva (realiza, abandonó y nunca ha realizado) y el consumo de alcohol (habitualmente, ocasionalmente, nunca y lo ha dejado). El tercer bloque de variables son las sociodemográficas 
entre las que se encuentra el sexo, la edad (de 15 a 29, de 30 a 44, de 45 a 59 y más de 60 años), los estudios realizados (sin estudios, Educación Primaria, Educación Secundaria Obligatoria y Post-obligatoria, Formación Profesional, Diplomados, y Licenciados) y la actividad principal (ama de casa, estudiante, trabajador privado, trabajador público, parado y jubilado-pensionista-rentista).

La aplicación del cuestionario se ha llevado a cabo durante los meses de febrero y marzo de 2003 aplicando el sistema de rutas aleatorias por secciones censales, lo que ha permitido seleccionar aleatoriamente la muestra dentro de cada sección censal.

\section{Análisis de datos}

En el estudio se han aplicado por un lado técnicas de análisis documental y, por otro, técnicas de análisis cuantitativo. Se hace imprescindible el análisis documental de otros estudios similares que permitan profundizar y contrastar los resultados obtenidos a través de fuentes secundarias. Después de haber sido tabulados y mecanizados informáticamente los datos, se ha procedido a su análisis cuantitativo, mediante el paquete de programas informático SPSS/PC (V 11.0), que ha posibilitado la creación de una base de datos sobre la cual se ha realizado el proceso de la creación de las redes bayesianas.

Actualmente, con el desarrollo de la tecnología los investigadores tienen a su disposición bases de datos que contienen un gran número de variables y de casos, que deberán ser procesadas para obtener información y sacar conclusiones. Una vía para estudiar los datos es mediante el ajuste de un modelo, es decir, el ajuste de una estructura que se supone que genera los datos. Una vez ajustado se utiliza para responder a las preguntas que son el objetivo del análisis de los datos.

Un modelo gráfico que se ha convertido en un área de investigación y aplicación en los últimos años son las redes bayesianas (Pearl, 1988), que son una herramienta útil para la representación de relaciones probabilísticas entre variables aleatorias y que han sido utilizadas con éxito en ámbitos muy diversos como en medicina (Andrassen, Jessen y Olensen, 1991; Beinlich, Suermondt, Chavez y Cooper, 1989; Mani, McDermott, y Valtorta, 1997), en diagnóstico de fallos (Chen y Talukdar, 1993), en predicción (Abramson, 1994; Gu, Peiris, Crawford, NcNicol, Marshall y Jefferies, 1994), en tutorización de alumnos (Conati y VanLehn, 1996), en biología (Friedman, Lineal, Nachman y Pe'er, 2000), en análisis de fiabilidad (Torres-Toledano y Sucar, 1998) o en pronóstico del tiempo (Kennett, Korb y Nicholson, 2001), entre otros.
Desde un punto de vista no formal, una red bayesiana constituye una representación gráfica de una distribución de probabilidad n-dimensional mediante un grafo dirigido y sin ciclos, en el cual cada nodo representa una variable de interés mientras que los arcos representan las probabilidades condicionales de relaciones de dependencia entre variables. Es decir, por un lado, se tiene la estructura de la red, que describe las relaciones de (in)dependencia condicional entre variables y, por otro, los parámetros de la red, que constituyen la parte cuantitativa de la distribución de probabilidad.

Las ventajas de representar una distribución de probabilidad mediante una red bayesiana son, entre otras, que permiten una comprensión rápida e intuitiva de las relaciones de (in)dependencia entre variables. A partir de las independencias condicionales que se pueden deducir en una red bayesiana se puede representar la distribución de probabilidad conjunta de una forma compacta. Aunque es necesario determinar las distribuciones condicionadas, este es un trabajo menos arduo que especificar la distribución conjunta.

Una vez que se ha construido una red bayesiana, algoritmos de propagación de la evidencia permiten que ésta sea utilizada para obtener información de variables de interés una vez que se introduce evidencia en según que otras variables que forman parte de la red.

\section{RESULTADOS}

Partiendo de un análisis comparativo donde se incluyen las variables consumo de alcohol y valoración de este consumo, en días laborables y fines de semana (tabla 2), donde consumo habitual es aquel que se realiza una o más veces por semana, ocasional el que se realiza alguna vez al mes, y quienes lo han dejado son aquellos que no han consumido en los últimos 12 meses, se obtiene que uno de cada dos almerienses afirma no haber bebido nunca alcohol (50\%) y uno de cada tres sólo de forma ocasional (34\%), si bien, hay un incremento del consumo durante los fines de semana que se explica al producirse un aumento de ocho unidades porcentuales entre los que consumen habitualmente y cuatro entre los que lo hacen ocasionalmente. De esta forma, el porcentaje de los que nunca consumen alcohol durante los fines de semana es menor, en doce unidades porcentuales, que aquellos que nunca lo hacen en días laborables.

Sucede lo mismo con la valoración que realizan los almerienses de su consumo, ya que son conscientes de que su ingesta de alcohol aumenta los fines de semana, al producirse un incremento de cinco unidades porcentuales con respecto a la media entre 
quienes dicen consumir mucho y un descenso en dieciséis unidades entre quienes dicen consumir casi nada o nada (tabla 2). con respecto a la media, incremento que es de cuarenta y cuatro unidades en los que valoran su consumo como poco (tabla 3). Esta situación es similar a la

Tabla 2. Consumo de alcohol y valoración en días laborables y fines de semana.

\begin{tabular}{|c|c|c|c|c|}
\hline & & Días laborables & Fines de semana & Incremento \\
\hline \multirow{4}{*}{ Consumo de alcohol } & Habitualmente & $7 \%$ & $15 \%$ & +8 \\
\hline & Ocasionalmente & $34 \%$ & $38 \%$ & +4 \\
\hline & Nunca & $50 \%$ & $38 \%$ & -12 \\
\hline & Lo ha dejado & $5 \%$ & $5 \%$ & 0 \\
\hline \multirow{3}{*}{$\begin{array}{c}\text { Valoración del } \\
\text { consumo de alcohol }\end{array}$} & Mucho & $2 \%$ & $7 \%$ & +5 \\
\hline & Poco & $27 \%$ & $38 \%$ & +11 \\
\hline & Nada o casi nada & $71 \%$ & $55 \%$ & -16 \\
\hline
\end{tabular}

Al considerar la relación que puede existir entre el consumo de alcohol y la valoración que ellos mismos hacen de su consumo, se halla una conexión directa entre ambas variables, tal y como se puede apreciar en la red bayesiana (figura 1), de modo que la probabilidad, en días laborables, de que un consumidor habitual de alcohol valore su consumo como mucho se incrementa sustancialmente en dieciséis unidades encontrada en los que dicen que nunca consumen, en quienes la probabilidad de que valoren su consumo como nada o casi nada es superior a veintitrés unidades, llegando a ser próximo al cien por cien, es decir, que la probabilidad de que una persona que dice no beber nunca valore su consumo como nada es de un noventa y cuatro por ciento (tabla 3).

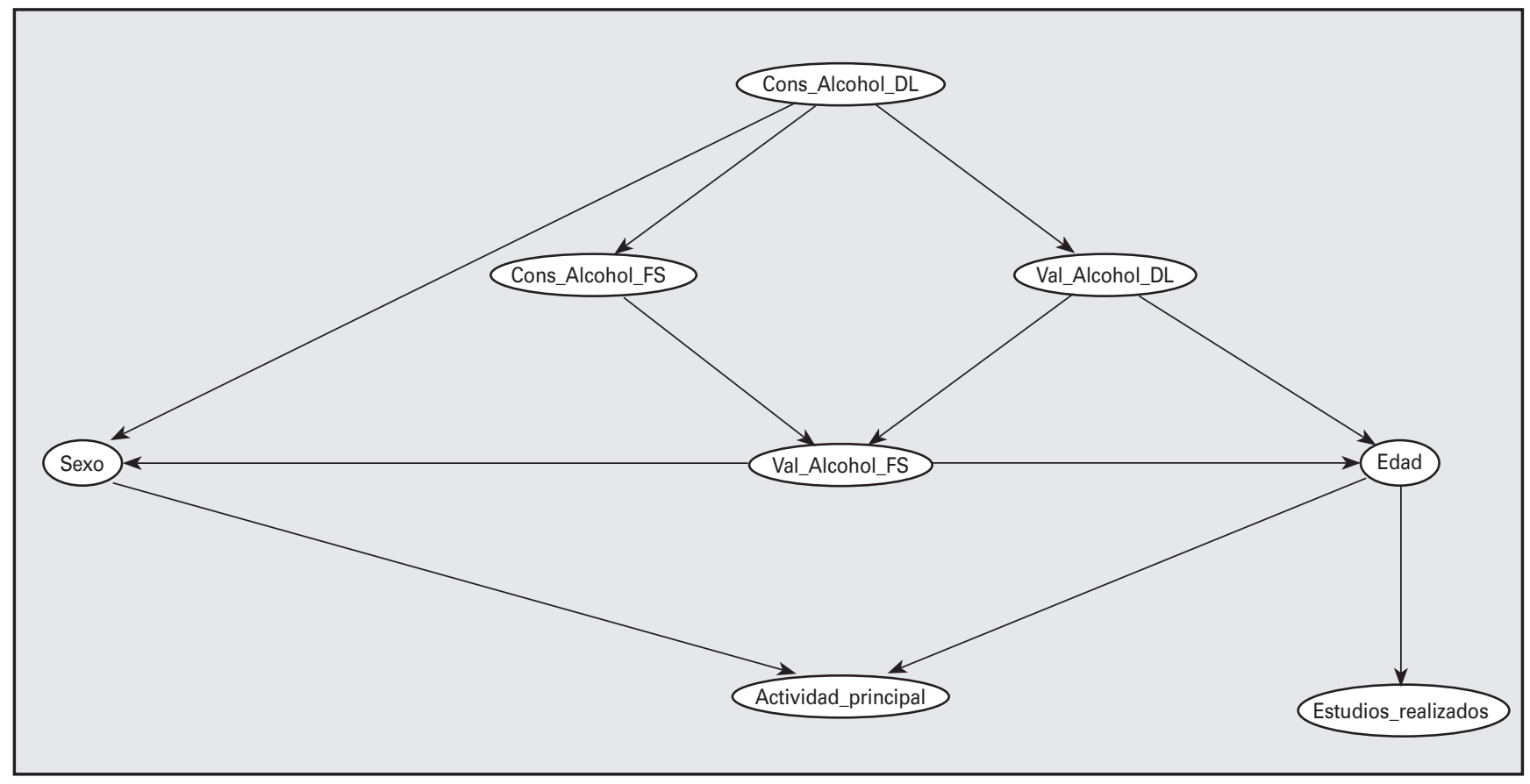

Figura 1. Relación entre el consumo y la autopercepción de consumo de alcohol con las variables sociodemográficas.

Leyenda:

Cons_Alcohol_DL: Alcohol consumido en días laborables.

Cons_Alcohol_FS: Alcohol consumido en fines de semana

Val_Alcohol_DL: Valoración del consumo de alcohol en días laborables.

Val_Alcohol_FS: Valoración del consumo de alcohol en fines de semana. 
Tabla 3. Probabilidades condicionales de relaciones de dependencia entre variables. Relación entre el consumo y la autopercepción de consumo de alcohol en días laborables y fines de semana.

\begin{tabular}{|c|c|c|c|c|c|}
\hline & & \multicolumn{4}{|c|}{ Consumo de alcohol } \\
\hline & & Media & Habitualmente & Ocasionalmente & Nunca \\
\hline \multirow{3}{*}{ Días laborales } & Mucho & $2 \%$ & $18 \%$ & $2 \%$ & $0 \%$ \\
\hline & Poco & $27 \%$ & $71 \%$ & $54 \%$ & $6 \%$ \\
\hline & Nada o casi nada & $71 \%$ & $12 \%$ & $44 \%$ & $94 \%$ \\
\hline \multirow{3}{*}{$\begin{array}{l}\text { Fines de sema- } \\
\text { na }\end{array}$} & Mucho & $7 \%$ & $32 \%$ & $5 \%$ & $1 \%$ \\
\hline & Poco & $38 \%$ & $57 \%$ & $67 \%$ & $7 \%$ \\
\hline & Nada o casi nada & $55 \%$ & $12 \%$ & $28 \%$ & $93 \%$ \\
\hline
\end{tabular}

Estas mismas circunstancias descritas se repiten en fines de semana, ya que en quienes beben de una manera habitual durante los fines de semana la probabilidad de que valoren su consumo como mucho es elevada, veinticinco unidades por encima de la media poblacional, al igual que en quienes valoran su consumo como poco, que aumenta en diecinueve unidades. Por su parte, los que dicen que nunca consumen, la probabilidad de que valoren su consumo como nada o casi nada es muy alta (93\%), es decir, treinta y ocho unidades por encima de la media. Al igual que sucede con los días laborables, quienes son consumidores ocasionales valoran su consumo como poco o nada, con veintisiete unidades por encima de la media.

Como se puede apreciar en la red bayesiana (figura 1), existe una relación directa entre el consumo de alcohol en días laborables y fines de semana, así como entre el consumo de alcohol y la valoración que se hace del mismo, produciéndose un incremento en la ingesta de alcohol en fines de semana con respecto a los días laborables, y una percepción de que se consume mucho alcohol entre quienes lo hacen de un modo habitual.

Si además del consumo de alcohol y su valoración se centra la atención en las variables sociodemográficas (sexo, edad, estudios realizados y actividad principal), se comprueba que existe una relación directa entre el consumo de alcohol en días laborables y el sexo de los almerienses (figura 1). También se puede observar que existe relación entre el consumo de alcohol durante los fines de semana y el sexo. Si bien esta relación no es directa, sino que están conectadas a través del consumo de alcohol durante los días laborables mediante conexiones divergentes, permite realizar inferencia entre ambas variables siempre que no se introduzca evidencia en la variable consumo de alcohol durante los días laborables (Jensen, 2001).
Así, utilizando la red de la figura 1 se observa que son las mujeres quienes tienen más probabilidad de no beber nunca (65\% los días de semana), y los varones de ser bebedores habituales y ocasionales, tanto en días laborables como en fines de semana $189 \%$ de forma habitual entre semana) (tabla 4).

La edad también guarda relación con el consumo de alcohol tanto en los días laborables como durante los fines de semana, aunque no de forma directa sino a través de otras variables (figura 1). En particular el consumo de alcohol durante los fines de semana está relacionado con la variable edad a través de la valoración durante el fin de semana y el consumo de alcohol durante los días laborables está relacionado con la variable edad a través de la valoración durante los días laborables, en ambos casos con una conexión del tipo en serie que permite realizar inferencia a través de dichas conexiones siempre que no se introduzca evidencia en la variable de conexión (Jensen, 2001). Así, podemos observar que son los menores de 45 años los que más beben (tabla 4), y en general manifestando la población ser consciente de este hecho ya que indican que su consumo es elevado, especialmente en fines de semana (tabla 2).

Otra variable relacionada de forma indirecta con el consumo de alcohol tanto en días laborables como en fines de semana es la variable sociodemográfica nivel de estudios, esta vez con conexiones en serie que incluyen dos variables, las comentadas anteriormente y la edad. Utilizando estas relaciones se comprueba que el sector que ha finalizado la primaria es el que más habitualmente consume alcohol entre semana (tabla 4), mientras que durante los fines de semana son los diplomados, junto con los estudiantes de secundaria, los que más alcohol toman. Los que carecen de estudios son quienes mayormente decla- 
Tabla 4. Probabilidades condicionales de relaciones de dependencia entre variables. Relación entre el consumo de alcohol entre semana con las variables sociodemográficas.

\begin{tabular}{|c|c|c|c|c|c|}
\hline & & \multicolumn{4}{|c|}{ Consumo de alcohol entre semana } \\
\hline & & Media & Habitualmente & Ocasionalmente & Nunca \\
\hline \multirow{2}{*}{ Sexo } & Varón & $49 \%$ & $89 \%$ & $62 \%$ & $35 \%$ \\
\hline & Mujer & $51 \%$ & $11 \%$ & $38 \%$ & $65 \%$ \\
\hline \multirow{4}{*}{ Edad } & De 15 a 29 años & $31 \%$ & $33 \%$ & $32 \%$ & $30 \%$ \\
\hline & De 30 a 44 años & $29 \%$ & $33 \%$ & $33 \%$ & $26 \%$ \\
\hline & De 45 a 59 años & $18 \%$ & $17 \%$ & $17 \%$ & $19 \%$ \\
\hline & Más de 59 años & $22 \%$ & $17 \%$ & $18 \%$ & $24 \%$ \\
\hline \multirow{6}{*}{$\begin{array}{l}\text { Nivel de } \\
\text { estudios }\end{array}$} & Sin estudios & $19 \%$ & $16 \%$ & $17 \%$ & $20 \%$ \\
\hline & Primarios & $42 \%$ & $42 \%$ & $42 \%$ & $41 \%$ \\
\hline & ESOY ESPO & $17 \%$ & $18 \%$ & $18 \%$ & $17 \%$ \\
\hline & Formación Profesional & $9 \%$ & $10 \%$ & $10 \%$ & $9 \%$ \\
\hline & Diplomatura & $8 \%$ & $8 \%$ & $8 \%$ & $7 \%$ \\
\hline & Licenciatura & $5 \%$ & $5 \%$ & $5 \%$ & $5 \%$ \\
\hline \multirow{6}{*}{$\begin{array}{l}\text { Actividad } \\
\text { principal }\end{array}$} & Parado & $6 \%$ & $5 \%$ & $6 \%$ & $6 \%$ \\
\hline & Estudiante & $13 \%$ & $14 \%$ & $14 \%$ & $13 \%$ \\
\hline & Ama de casa & $17 \%$ & $5 \%$ & $13 \%$ & $22 \%$ \\
\hline & Trabajador privado & $33 \%$ & $41 \%$ & $36 \%$ & $29 \%$ \\
\hline & Trabajador público & $14 \%$ & $18 \%$ & $16 \%$ & $12 \%$ \\
\hline & Jubilado/pensionista/rentista & $16 \%$ & $17 \%$ & $16 \%$ & $17 \%$ \\
\hline
\end{tabular}

ran no haber consumido nunca alcohol $(20 \%$ en días laborables).

Igualmente la actividad principal está relacionada con el consumo de alcohol de forma indirecta (figura 1). Al analizar la ingesta de alcohol según la actividad principal, se desvela que es el colectivo de parados quien más realiza un consumo habitual, tanto en días laborables como en fines de semana. Los trabajadores del sector público son los que con mayor frecuencia consumen bebidas alcohólicas de manera ocasional, en ambos momentos de la semana. Las amas de casa y los estudiantes son quienes más declaran no haber tomado nunca alcohol, durante la semana, desligándose en los fines de semana el colectivo de estudiantes, para ser las amas de casa las que presentan los valores más altos (tabla 4).

Descrito el comportamiento de estas variables, interesa conocer el perfil del ciudadano almeriense que consume de un modo habitual y aquellos que nunca han ingerido alcohol, para lo cual se ha vuelto a recurrir a la red bayesiana (figura 1), obteniendo que los almerienses que son consumidores habituales de alcohol y, por tanto, poblaciones en riesgo para la salud, son los varones que tienen entre 15 y 44 años, que poseen estudios secundarios o con una licenciatura y que trabajan en el sector público o privado. Aquellos que dicen ser bebedores ocasionales son los varones, menores de 44 años, que trabajan en el sector público y privado y que poseen la secundaria o unos estudios superiores. En el lado opuesto, es decir, aquellos que no consumen nada de alcohol se encuentran las mujeres que tienen más de 44 años, que carecen de estudios y que son amas de casa.

Conocido el comportamiento de las variables sociodemográficas en el consumo de alcohol, se plantea interesante descubrir que relación puede existir entre la ingesta de alcohol con las variables autopercepción de salud, autopercepción de la condición física, práctica e interés por la actividad físico-deportiva durante el tiempo libre. Tras elaborar una nueva red bayesiana, donde se han incluido estos factores (figura 2), se puede apreciar como existe una relación entre estas variables. Utilizando esta red de forma similar a la de la figura 1, se obtiene que son los consumidores habituales de alcohol, quienes muestran un mayor interés por la actividad físico-deportiva, son practicantes en su tiempo libre o han abandonado la práctica físicodeportiva y tienen una buena autopercepción de su condición física y de su salud. En cambio, en quienes indican que nunca beben alcohol, se obtiene una mayor probabilidad de no haber practicado nunca actividad físico-deportiva, y poseer una autopercepción regular de su salud (tabla 5). 


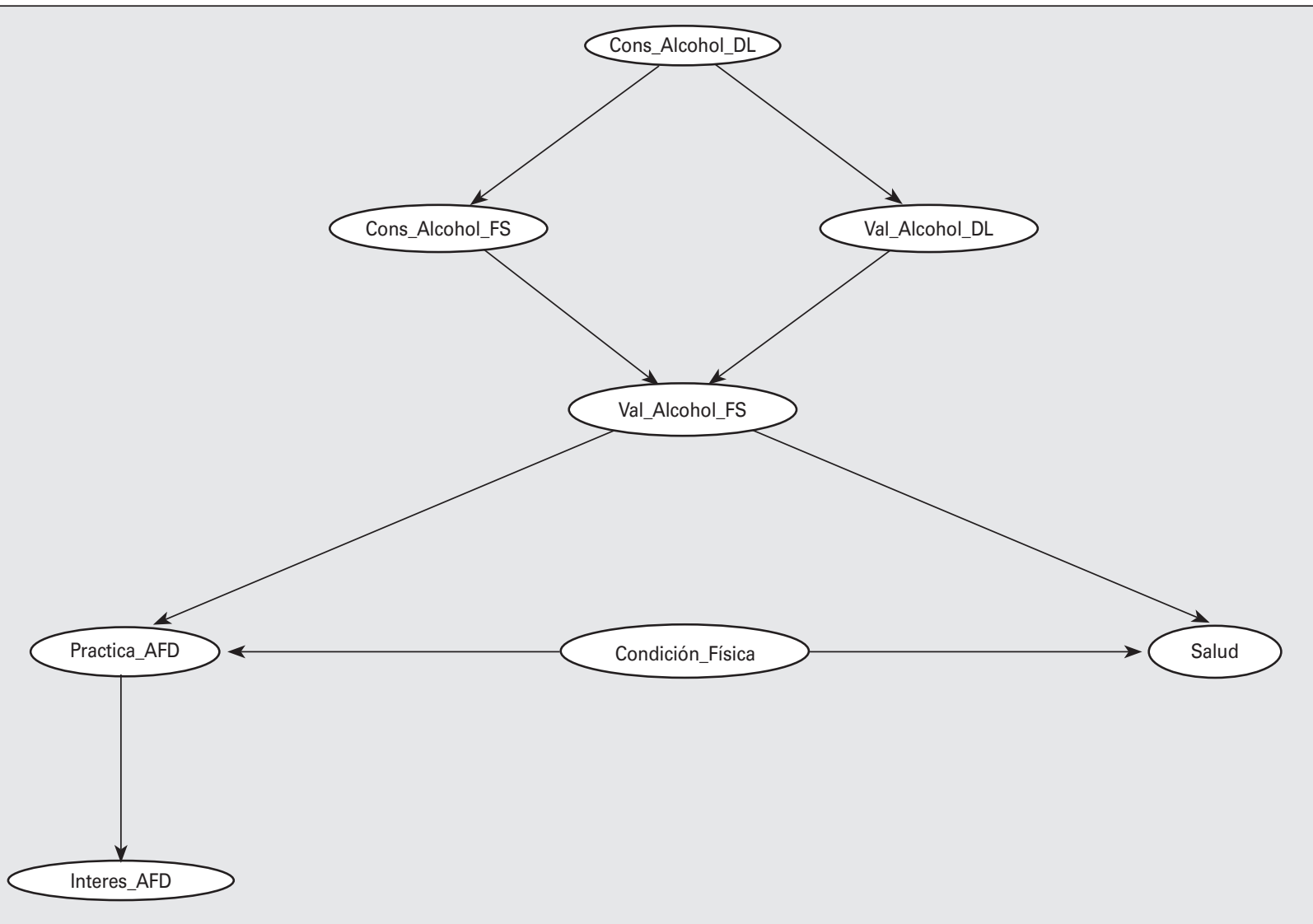

Figura 2. Relación entre el consumo y la autopercepción de consumo de alcohol con variables relacionadas con la actividad físico-deportiva y la autopercepción de la salud.

Leyenda:

Cons_Alcohol_DL: Alcohol consumido en días laborables.

Cons_Alcohol_FS: Alcohol consumido en fines de semana.

Val_Alcohol_DL: Valoración del consumo de alcohol en días laborables.

Val_Alcohol_FS: Valoración del consumo de alcohol en fines de semana.

Interes_AFD: Interés por la actividad físico-deportiva.

Condición_Física: Percepción de Condición Física.

Salud: Percepción de Salud.

Práctica_AFD: Actividad físico-deportiva practicada.

Tabla 5. Probabilidades condicionales de relaciones de dependencia entre variables. Relación entre el consumo de alcohol con variables relacionadas con la actividad físico-deportiva y la autopercepción de la salud.

\begin{tabular}{|c|c|c|c|c|c|}
\hline & & \multicolumn{4}{|c|}{ Consumo de alcohol } \\
\hline & & Media & Habitualmente & Ocasionalmente & Nunca \\
\hline \multirow{2}{*}{ Interés AFD } & Alto & $51 \%$ & $54 \%$ & $52 \%$ & $49 \%$ \\
\hline & Bajo & $49 \%$ & $45 \%$ & $47 \%$ & $50 \%$ \\
\hline \multirow{3}{*}{ Práctica AFD } & Practicantes & $37 \%$ & $42 \%$ & $40 \%$ & $34 \%$ \\
\hline & Abandonaron & $29 \%$ & $33 \%$ & $31 \%$ & $28 \%$ \\
\hline & Nunca realizaron & $34 \%$ & $25 \%$ & $29 \%$ & $38 \%$ \\
\hline \multirow{3}{*}{ Condición física } & Buena & $58 \%$ & $62 \%$ & $61 \%$ & $56 \%$ \\
\hline & Regular & $31 \%$ & $29 \%$ & $30 \%$ & $32 \%$ \\
\hline & Mala & $10 \%$ & $9 \%$ & $8 \%$ & $11 \%$ \\
\hline \multirow{3}{*}{ Salud } & Buena & $69 \%$ & $76 \%$ & $74 \%$ & $65 \%$ \\
\hline & Regular & $26 \%$ & $20 \%$ & $23 \%$ & $29 \%$ \\
\hline & Mala & $5 \%$ & $4 \%$ & $3 \%$ & $6 \%$ \\
\hline
\end{tabular}




\section{DISCUSIÓN}

La prevalencia de consumo de alcohol entre la población almeriense en días laborables (41\%), está por debajo de la media andaluza $(53,4 \%)$ (Junta de Andalucía, 1997) y de la media nacional $(51,8 \%)$ (Plan Nacional sobre Drogas, 2005), produciéndose un incremento durante los fines de semana (53\%) a pesar del cual se sigue manteniendo por debajo de la media nacional (63,8\%). La valoración global que se desprende del consumo de alcohol entre semana es positiva, si se tiene en cuenta que la cifra de consumidores habituales se halla por debajo del 10\%, frente a la prevalencia de consumo diario de la media nacional que se sitúa en el 14,1\% (Plan Nacional sobre Drogas, 2005). El ciudadano es consciente del consumo que realiza de esta sustancia, pues tanto entre semana como en fines de semana, tiene una autopercepción en consonancia con su comportamiento, al establecerse una conexión directa entre consumo de alcohol y autovaloración, siendo aquellos que ingieren habitualmente alcohol, quienes perciben que beben mucho, y quienes nunca ingieren los que valoran que beben casi nada o nada. Esta percepción acertada de los almerienses hacia su consumo podría estar vinculada con una adecuada percepción del riesgo y los posibles problemas que puede acarrear, lo que explicaría una ingesta más moderada del alcohol de la población almeriense con respecto a la media nacional (Plan Nacional sobre Drogas, 2005).

Si bien no son numerosas las investigaciones que distinguen entre el consumo de alcohol en días laborables y fines de semana, las que así lo contemplan obtienen resultados en la misma línea de Simpson et al. (2002), informando de un consumo mayor del $75 \%$ y del $67 \%$, en varones y mujeres respectivamente, durante las noches de los fines de semana. Resultados en la misma línea son expuestos en el Plan Nacional sobre Drogas (2005), obteniendo una prevalencia en el consumo de alcohol en fines de semana del $75.5 \%$ en hombres y del $51.7 \%$ en mujeres. Pascual (2002a), añade que esta práctica se centra especialmente en la población adolescente que busca los efectos de embriaguez, en lugares de diversión y en la calle, junto con sus compañeros de grupo y fuera del ámbito del hogar, coincidiendo con los datos arrojados por la Junta de Andalucía (1997), así como con el incremento en fines de semana. Estudios realizados a nivel nacional, como los de Camacho, Manzanares y Guillén (2002), y Duque, Terrón, López, Sánchez y Romero (1995), muestran que el $70 \%$ de los adolescentes manifiestan haber probado el alcohol y más de un $30 \%$ de manera habitual, quedando claramente identificado que es este sector de la población quien realiza un mayor consumo.

A través de las redes bayesianas se ha podido hallar una relación además del consumo de alcohol con la edad, también con el sexo, ya que son los varones quienes más consumen frente a las mujeres y este consumo habitual es más frecuente entre los más jóvenes. Esto viene a indicar una tendencia en consonancia con los resultados obtenidos en el estudio de Gómez-Talegón et al. (2005), donde analizan la evolución del consumo de alcohol de los españoles entre 1993 y 1997, a partir de los datos de la encuesta nacional de salud, y resaltan que son los varones quienes más ingieren este tipo de droga y especialmente entre el sector de la población joven.

En el informe del 2004 del Observatorio Español sobre Drogas (Plan Nacional sobre Drogas, 2005), se constata un consumo más extendido entre hombres, así como una práctica mucho más extendida de emborracharse. Por el contrario, son las mujeres en quienes se encuentra la mayor probabilidad de no consumir nada de alcohol, tal y como manifiestan Denton y Walters (1999), en un trabajo en el que analizan las variables que promueven los hábitos de vida saludables, este hábito es menos aceptable en el sector femenino frente a los hombres que es más probable que fumen y beban.

En esta investigación se ha tratado principalmente de conocer la relación entre la práctica de actividad físico-deportiva y el consumo de alcohol, obteniendo que lo más probable es que las personas que realizan una práctica físico-deportiva regular sean varones, consumidores habituales y ocasionales de esta sustancia, teniendo un elevado interés por la actividad físico-deportiva y una buena autopercepción tanto de su salud como de su condición física. En el lado opuesto, se hallan las mujeres que no consumen nada de alcohol, que declaran que nunca han practicado ejercicio físico y que poseen una autopercepción de su salud regular. Estos resultados están en sintonía con los obtenidos por Andradas y Fernández (1994), en la comunidad de Madrid, que indican que los varones únicamente tienen unos hábitos saludables frente a las mujeres en cuanto a la práctica del deporte, mientras que el colectivo femenino tiene mejores hábitos de salud en la alimentación, higiene, consumo de tabaco y alcohol, y ocupación del tiempo libre, a pesar de poseer una peor percepción de su condición física. Una posible explicación al hecho de que sean precisamente las personas que realizan actividad física de forma regular y poseen una buena percepción de su salud, las que también realizan un consumo habitual y ocasional de esta sustancia, es la asociación que puede llegarse a establecer entre ejercicio físico y efectos beneficiosos que reporta sobre la salud, lo que les genera una mayor confianza, sintiendo que su práctica de actividad-físico-deportiva es un método eficaz para contrarrestar los efectos perniciosos que provoca el alcohol. 
Analizando con detenimiento los resultados de los distintos estudios que tratan de encontrar relación entre el consumo de alcohol y la práctica de actividad físico-deportiva, obtenemos que los hallazgos son realmente contradictorios ya que, por un lado, se encuentran investigaciones como la de Moral, Rodríguez y Sirvent (2005), que destacan la evitación de problemas de salud física como uno de los principales motivos por los que no beber alcohol y, en cambio, otros estudios como el de Nebot, Comín, Villalba y Murillo (1991), descubren una relación positiva entre la opinión que tienen hacia el deporte, la salud y el consumo de alcohol. Aquellos que son físicamente activos son quienes mejor perciben su salud, y por tanto, es posible que también sean quienes más confiados se puedan sentir de consumir alcohol, sin que repercuta negativamente sobre su percepción general de condición física y salud.

El deporte ha de ayudar al niño en su desarrollo, en su educación y en su aprendizaje tal como apunta Pelegrín (2002), en su trabajo sobre conducta agresiva y deporte, en cambio no parece que los programas de actividad físico-deportiva ofertados a los jóvenes para contrarrestar los hábitos de consumo de alcohol, especialmente en fines de semana, sean realmente eficaces, entre otros motivos porque la falta de actividad física según Bourdeaudhuij y Van Oost (1999), no es uno de los factores de riesgo en los programas de educación, diseñados para cambiar el comportamiento de hábitos relacionados con la salud.

Futuras investigaciones se hacen necesarias para comprobar el efecto que produce la práctica de actividad físico-deportiva como medida preventiva ante el consumo de sustancias alcohólicas, como así también lo señalan Bourdeaudhuij y Van Oos (1999) o Paavola et al. (2004), en sus respectivos estudios. Los consumidores habituales de alcohol poseen una buena autopercepción de su condición física y de su salud, por lo que, entre las posibles soluciones para reducir y prevenir la ingesta de alcohol, se ha de reorientar el motivo de práctica físico-deportiva de la sociedad hacia el interés por lograr un mayor grado de bienestar, en lugar de una mejor forma física o un estado de salud más elevado, tal y como señala Schmohr, Kristensen, Prescott y Scharling (2005), dirigiendo la atención de la población hacia el incremento del bienestar como pieza clave de las campañas de prevención con el propósito de aumentar el tiempo de ocio dedicado a la actividad física, a lo que Portero et al. (2002), añaden sustituir el término prevención por el de mantener, mejorar o recuperar la salud, para así implicar la idea de bienestar físico y psíquico.

Consideramos de especial interés, en próximos estudios contemplar las relaciones que se establecen entre las variables actividad físico-deportiva y consumo de alcohol, en función del sexo y de la edad, ana- lizando por separado a los varones y a las mujeres, y su comportamiento en los distintos sectores de edad, ya que probablemente el patrón de comportamiento habitual en las mujeres de mayor edad es que no consuman alcohol y que tampoco hagan ejercicio físico.

Por último, resaltar como principales conclusiones de este estudio un incremento de la ingesta de alcohol en fines de semana, acompañado de una mayor percepción del consumo de esta sustancia. Existe una conexión directa entre la ingesta de alcohol con el sexo y la edad de los sujetos, además de una relación positiva entre el consumo de esta sustancia y la práctica e interés por la actividad físico-deportiva. Lo más probable es que las personas que realizan una práctica físico-deportiva regular sean varones y jóvenes, que poseen estudios secundarios o una licenciatura, que trabajan en el sector público o privado, consumidores habituales de esta sustancia, que tienen un elevado interés por la actividad físico-deportiva y una buena autopercepción tanto de su salud como de su condición física. Mientras que son las mujeres, con más de 44 años, amas de casa, quienes declaran que nunca han practicado ejercicio físico y que poseen una autopercepción de su salud regular, a pesar de no consumir nada de alcohol.

\section{AGRADECIMIENTOS}

Este estudio ha sido financiado por el Consejo Superior de Deportes y catalogado de alto impacto según expediente 16/UPB/2001 (resolución 10.7 de 24 de 2001, BOE n 118 y 17/05/01, y expediente 07/UPB20/04 (resolución 29/01/2004, BOE nº 62 y 12/03/04). También ha recibido una subvención del Instituto de Estudios Almerienses para el curso 20032004.

\section{REFERENCIAS}

Abramson, B. (1994). The design of belief network-based Systems for price forecasting. Computers and Electrical Engineering, 20, 163-180.

Andradras, V. y Fernández, M. I. (1994). Hábitos de salud de los escolares de una zona periurbana de Madrid. Revista de Sanidad e Higiene Pública, 68, 203-212.

Andrassen, S., Jessen, F. y Olensen, K. (1991). Medical expert systems based on probabilistic causal networks. International Journal of Medical Computing, 28, 1-30.

Balaguer, I., Pastor, Y. y Moreno, Y. (1999). Algunas características de los estilos de vida de los adolescentes de la Comunidad Valenciana. Revista Valenciana D'Estudis Autonómics, 26, 33-56. 
Beinlich, I. A., Suermondt, H. J., Chavez, R. M. \& Cooper, G. F. (1989). The ALARM monitoring system: A case study with two probabilistic inference techniques for belief networks. En: Proceedings of the Second European Conference on Artificial Intelligence in Medicine, pp. 247-256.

Blair, S.N., Jacobs, D.R. y Powell, K.E. (1985). Relationships between exercise or physical activity and other health behaviors. Public Health Reports, 100, 172-180

Bourdeaudhuij, I. y Van Oost, P. (1999). A cluster-analytical approach toward physical activity and other health related behaviours. Medicine \& Science in Sports \& Exercise, 31, 605-612.

Buendía, L. (1998). El proceso de investigación. En M. P. Colás Bravo y L. Buendía Eximan, Investigación educativa (pp. 69-105). Sevilla: Alfar.

Camacho, A., Manzanares, M. y Guillén, M. (2002). Estudio sobre indicadores de salud relacionados con la actividad física en escolares de 12 a 16 años. Lecturas Educación física y Deportes, Revista Digital, 54. Recuperado el 27 de enero de 2005, de http://www.efdeportes.com

Carrasco, A.M. (2004). Consumo de alcohol y estilos de vida: una tipología de los adolescentes españoles. Revista de Psicología Social, 19, 51-79.

Chen, C. L. y Talukdar, S. (1993). Causal nets for diagnosis (of power systems). En: Expert Systems Application to Power Systems IV Proceedings, pp. 379-386.

Conati, C. y VanLehn, K. (1996). Probabilistic plan recognition for cognitive apprenticeship, En: Proceedings of the $18^{\text {th }}$ Annual Conference of the Cognitive Science Society (pp. 403-408). San Diego, CA,

Denton, M. y Walters, V. (1999). Gender differences in structural and behavioral determinants of health: an analysis of the social production of health. Social Science \& Medicine, 48, 1221-1235.

Duque, G., Terrón, I., López, M. A., Sánchez, J. y Romero, M. P. (1995). Consumo de tabaco, alcohol y drogas ilegales entre alumnos de BUP. Salud Rural, 6, 49-57.

Friedman, N., Linial, M., Nachman, I. y Pe'er, D. (2000). Using Bayesian networks to analyze expression data. Journal of Computational Biology 7, 601-620.

García Ferrando, M. (2000). La encuesta. En M. García Ferrando, J. Ibáñez, y F. Alvira (Eds.), El análisis de la realidad social. Métodos y técnicas de investigación (pp. 167-201) (3 ${ }^{\mathrm{a}}$ ed.). Madrid: Alianza Editorial.

Gómez-Talegón, M. T., Prada, C., Río, M. C. y Álvarez, F. J. (2005). Evolución del consumo de alcohol de los españoles entre 1993, 1995 y 1997, a partir de los datos de la encuesta nacional de salud. Adicciones, 17, 17-27.

Gu, Y., Peiris, D. Crawford. J. NcNicol. J., Marshall, B., y Jefferies, R. (1994). An application of belief networks to future crop production. En: Proceedings of the 10th Conference on Artificial Intelligence for Application IEEE (pp. 305-309).Computer Society Press.

Hidalgo, I., Garrido, G. y Hernández, M. (2000). Health status and risk behavior of adolecents in the North of Madrid, Spain. Journal of Adolescent Health, 27, 351-360.
Junta de Andalucía (1997). Los andaluces ante las drogas. Sevilla: Consejería de Asuntos Sociales.

Jensen, F.V. (2001). Bayesian networks and decisión graphs. New York: Springer-Verlang.

Kennett, R., Korb, K. y Nicholson, A. (2001). Seabreeze prediction using Bayesian networks: A case of study. En: Proceedings of the $5^{\text {th }}$ Pacific-Asia Conference on Advances in Knowledge Discovery and Data Mining (pp. 148-153). New York: Springer-Verlang,

Mani, S.; McDermott, S. Valtorta, M. (1997). Mentor: A Bayesian model for prediction of mental retardation in newborns. Research in Developmental Disabilities, 18, 303-318.

Martínez, R. (1995). Psicometría: Teoría de test psicológicos y educativos. Madrid: Síntesis.

Martínez, J. M. y Robles, L. (2001). Variables de protección ante el consumo de alcohol y tabaco en adolescentes. Psicothema, 13, 222-228.

Mora-Rios, J. y Natera, G. (2001). Expectativas, consumo de alcohol y problemas asociados en estudiantes universitarios de la ciudad de México. Salud Pública de México, 43, 89-96.

Moral, M. V., Rodríguez, F. J. y Sirvent, C. (2005). Motivadores de consumo de alcohol en adolescentes: análisis de diferencias inter-género y propuesta de un continuum etiológico. Adicciones, 17, 105-120.

Nebot, M., Comín, E., Villalba, J. R. y Murillo, C. (1991). La actividad física de los escolares: un estudio transversal. Revista de Sanidad e Higiene Pública, 65, 325-331.

Paavola, M., Vartiainen, E. y Haukkala, A. (2004). Smoking, Alcohol Use, and Physical Activity: A 13-Year Longitudinal Study Ranking from Adolecence into Adulthood. Journal of Adolescent Health, 35, 238-244.

Pascual, F. (2002a). Imagen social de las bebidas alcohólicas. Adicciones, 14, 115-122.

Pascual, F. (2002b). Percepción del alcohol entre los jóvenes. Adicciones, 14, 123-131.

Pastor, Y., Balaguer, I. y García-Merita, M. (2006). Relaciones entre el autoconcepto y el estilo de vida saludable en la adolescencia media: un modelo exploratorio. Psicothema, 18, 18-24.

Pate, R.R., Heath, G.W., Dowda, M. y Trost, S.G. (1996). Associations between physical activity and other health behaviours in a representative sample of US adolescents. American Journal of Public Health, 86, 1577-1581.

Pearl, J. (1988). Probabilistic Reasoning in Expert Systems: Networks of plausible inference. San Francisco, CA: Morgan Kaufmann.

Pelegrín, A. (2002). Conducta agresiva y deporte. Cuadernos de Psicología del Deporte, 2, 39-56.

Plan Nacional sobre Drogas (1998). Informe $n^{0} 1$. Observatorio Español sobre Drogas. Madrid: Ministerio del Interior.

Plan Nacional sobre Drogas (2005). Informe 2004 del Observatorio Español sobre Drogas. Situación y tendencias de los problemas de drogas en España. Madrid: Ministerio del Interior. 
Portero, P., Cirne, R. y Mathieu, G. (2002). La intervención con adolescentes y jóvenes en la prevención y promoción de la salud. Revista Española de Salud Pública, 76, 577-584.

Riala, K., Hakko, H., Isohanni, M., Järvelín y Räsänen, P. (2004). Teenage Smoking and Substance Use as Predictors of Severe Alcohol Problems in Late Adolescente and in Young Adulthood. Journal of adolescent health, 35, 245-254.

Ruiz Juan, F. y García Montes, M. E. (2005). Significado del estudio, objetivos y metodología. En: F. Ruiz Juan y M. E. García Montes (Eds.), Hábitos físico-deportivos de los almerienses en su tiempo libre (pp. 1-22). Almería: Servicio Publicaciones de la Universidad de Almería.

Santo-Domingo, J. (2002). El desarrollo personal del joven y el alcohol. Trastornos Adictivos, 4, 223-232.
Schmohr, P., Kristensen, T.S., Prescott, E. y Scharling, H. (2005). Stress and life dissatisfaction are inversely associated with jogging and other types of physical activity in leisure time - The Copenhagen City Heart Study. Scandinavian Journal of Medicine \& Science in Sports. 15, 107-112.

Simpson, W.F., Brehm, H.N., Rasmussen, M.L., Ramsay, J. y Probst, J.C. (2002). Health and fitness profiles of collegiate undergraduate students. Journal of Exercise Physiology, 5, 14-27.

Torres-Toledano, J. G. y Sucar, L.E. (1998). Bayesian networks for reliability analysis of complex systems. En: Progress in Artificial Intelligence-IBERAMIA 98 (pp.195-206). Berlín: Springer. 